\title{
Pushing the frontier of WIMPy inelastic dark matter: Journey to the end of the periodic table
}

\author{
Ningqiang Song $\odot,^{1,2,3, *}$ Serge Nagorny $\odot,^{1,2, \dagger}$ and Aaron C. Vincent ${ }^{1,2,3, \sharp}$ \\ ${ }^{1}$ Department of Physics, Engineering Physics and Astronomy, Queen's University, \\ Kingston, Ontario K7L 3N6, Canada \\ ${ }^{2}$ Arthur B. McDonald Canadian Astroparticle Physics Research Institute, Department of Physics, \\ Engineering Physics and Astronomy, Queen's University, Kingston, Ontario K7L 3N6, Canada \\ ${ }^{3}$ Perimeter Institute for Theoretical Physics, Waterloo, Ontario N2L 2Y5, Canada
}

(Received 27 April 2021; accepted 5 November 2021; published 30 November 2021)

\begin{abstract}
We explore the reach of low-background experiments made of small quantities of heavy nuclear isotopes in probing the parameter space of inelastic dark matter that is kinematically inaccessible to classic direct detection experiments. Through inelastic scattering with target nuclei, dark matter can yield a signal via either nuclear recoil or nuclear excitation. We present new results based on this approach, using data from low-energy gamma quanta searches in low-background experiments with Hf and Os metal samples and measurements with $\mathrm{CaWO}_{4}$ and $\mathrm{PbWO}_{4}$ crystals as scintillating bolometers. We place novel bounds on WIMPy inelastic dark matter up to mass splittings of about $640 \mathrm{keV}$ and provide forecasts for the reach of future experiments.
\end{abstract}

DOI: $10.1103 /$ PhysRevD.104.103032

\section{INTRODUCTION}

Dark matter (DM) direct detection experiments are designed to record the small amounts of energy expected to be deposited by galactic DM particles as they pass through the detector and interact with nuclei or electrons in the target material. Most such detectors are optimized to search for WIMP-like particles, with masses in the GeV to $\mathrm{TeV}$ range, and weak-scale DM-nucleon elastic scattering cross sections $\sigma_{n} \lesssim 10^{-40} \mathrm{~cm}^{2}$. These typically employ target nuclei such as $\mathrm{C}, \mathrm{O}, \mathrm{Si}, \mathrm{Ar}, \mathrm{Ge}$, or $\mathrm{Xe}$, which are kinematically well matched to the $10-100 \mathrm{GeV}$ range. Experiments are typically conducted deep underground to mitigate cosmic-ray and radiogenic background components.

However, evidence for the existence of DM on large scales does not directly constrain its mass range or interaction strength but rather its mass density (about 0.3 times the critical density $\rho_{c}$ on large scales and around $0.4 \mathrm{GeV} \mathrm{cm}^{-3}$ in the Solar neighborhood) and interaction rate with the Standard Model (SM) of particle physics.

\footnotetext{
*ningqiang.song@queensu.ca

†n65@queensu.ca

*aaron.vincent@queensu.ca
}

Published by the American Physical Society under the terms of the Creative Commons Attribution 4.0 International license. Further distribution of this work must maintain attribution to the author(s) and the published article's title, journal citation, and DOI. Funded by SCOAP ${ }^{3}$.
As the viable parameter space for WIMP-like dark matter shrinks following null measurements from sensitive direct detection experiments developed over the past decades, the scope of DM direct detection searches has broadened. Candidates include sub-GeV dark matter [1-12], superheavy dark matter [13-19], and macro dark matter [20-23]. However, the weakly interacting massive particle (WIMP) family still includes many viable candidates that remain out of reach from the current generation of experiments. In particular, inelastic (I)DM particles, with $O(\mathrm{keV})$ or larger mass splittings, are expected in a variety of dark matter models [24-33]. Multistate DM has been invoked in possible explanations of the $511 \mathrm{keV}$ gamma-ray excess observed in the Galactic center [34-38] and the DAMA/ LIBRA annual modulation signals [25,39-42]. Recently, interest in inelastic dark matter has also been revived to interpret the XENON1T electron recoil data [43] with exothermic scattering or luminous dark matter [44-59].

A simple inelastic dark matter model consists of a lighter state $\chi_{1}$ and a heavier state $\chi_{2}$ with a mass splitting $\delta \equiv M_{\chi_{2}}-M_{\chi_{1}}$. Generically, the lighter state will dominate the relic abundance, and two-to-two scattering processes with baryons must lead to an excitation or deexcitation of the DM state. In contrast to elastic scattering, the kinematics of inelastic dark matter exhibits two features. First, the dark matter kinetic energy must be large enough to overcome the mass splitting, imposing a lower limit on the relative velocity required for an interaction to take place. Second, the minimum momentum exchange required for a collision to occur increases with the mass splitting, 
meaning that IDM with typical halo velocities can scatter only with heavier nuclei and deposit large enough recoil energy. In other words, even with sufficient kinetic energy, there is a momentum transfer threshold $\sim \mu_{\gamma N} v$ below which interactions may not occur. As a consequence, inelastic dark matter may evade the searches in most direct detection experiments, either because the analysis region is limited to low $(\lesssim 10-50 \mathrm{keV})$ recoil energies or because of the limited target nucleus mass.

We, thus, turn to heavier target nuclei $(Z>54)$, which have received little attention in the search for DM-induced recoils. Many elements between $Z \sim 58$ (cerium) and $Z \sim 83$ (bismuth) are sensitive to IDM models with $\gtrsim 100 \mathrm{keV}$ mass splittings while remaining sufficiently stable not to overwhelm a detector with alpha or beta decay backgrounds. Such elements, in particular, tungsten and lead, have been utilized in scintillating crystals to search for rare alpha decays $[60,61]$. These experiments feature both light and heat measurements, which provide a powerful tool to distinguish DM-induced nuclear recoil (NR) events due to high momentum-transfer coherent DMnucleus interactions (Fig. 1, top) from background events.

In addition to nuclear recoil signals from coherent scattering, heavier elements can contribute a second type of signal, thanks to their comparatively loosely bound outer
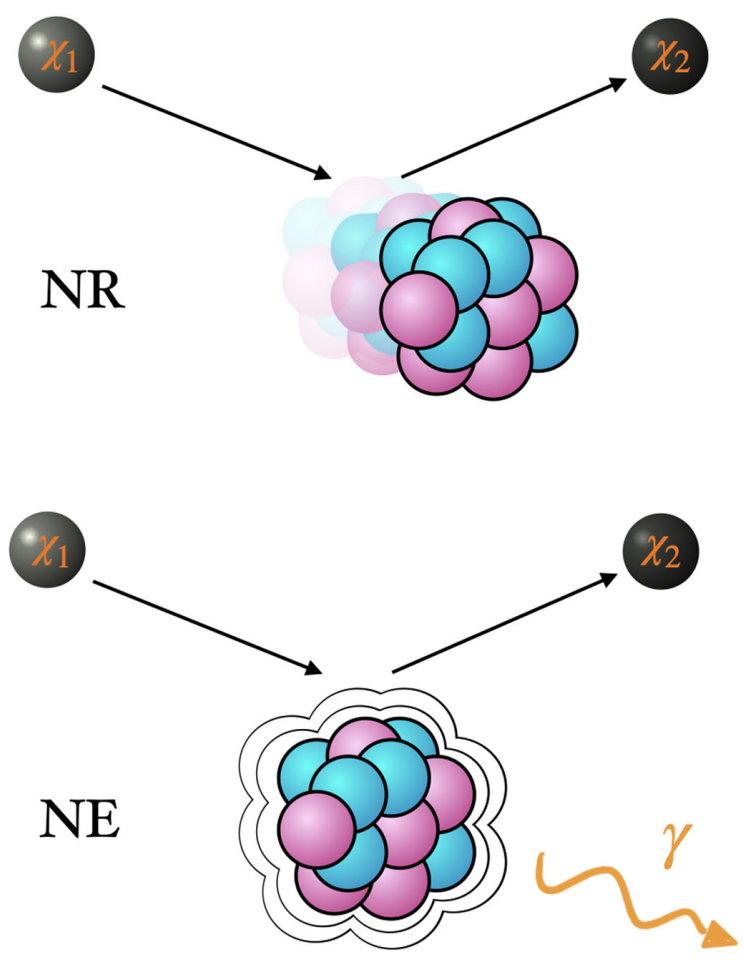

FIG. 1. The two processes considered in this work. Nuclear recoil (NR): Dark matter is upscattered to a heavier state and the target nucleus recoils from the impact, leading to heat, scintillation, and/or ionization signals. Nuclear excitation (NE): In addition to NR, the nucleus can also be excited and then deexcite to ground state with the emission of one or several gamma quanta. nucleon shells: gamma photons from induced nuclear excitation (NE) (Fig. 1, bottom), and subsequent decay, or deexcitation of a long-lived excited nuclear state. The goal of this paper is, thus, to use these two approaches, i.e., nuclear recoil searches and nuclear transition gammas, to place novel constraints on inelastic DM models.

Recently, a search for collisional deexcitation of the metastable nuclear isomer ${ }^{180 m} \mathrm{Ta}$ induced by DM has led to novel constraints on IDM $[62,63] .{ }^{180 m} \mathrm{Ta}$ is fairly unique: It is stable on timescales much larger than the age of the Universe, and its natural abundance is well established. As the isomers of other nuclei are short-lived, this method is not generally applicable. However, the excitation of ground state nuclei to excited states remains possible in the scattering $\chi_{1}+N \rightarrow \chi_{2}+N^{*}$ if the excitation energy is low enough [64-66]. We focus on a few isotopes, with possible nuclear transitions that we will explore listed in Table I below. Once scattered into an excited state, the gamma quanta emitted during subsequent decay to the ground state can be measured. By measuring the activity of these transition lines, we may obtain a conservative upper bound on the total inelastic scattering rate from DM interactions and, thus, on the DM-nucleon scattering cross section. In comparison with traditional direct detection experiments, this method appears rather insensitive. Indeed, nuclear excitation is more kinematically suppressed, and the scattering does not enjoy the usual $A^{2}$ enhancement factor, because the DM is interacting with a single nucleon rather than scattering coherently with the full nucleus. However, we identify two advantages: (i) The high mass of transition nuclei better matches heavier DM candidates, and (ii) the energy of the outgoing gamma is independent of the recoil energy, since it is fixed by the structure of the target nucleus. This means that, for a given transition, there is no danger of the signal being lost outside of the analysis region of interest. Previously, we have performed such an analysis in the context of a search for rare decays of Hf [67] and set stronger bounds on IDM mass splittings than previously reported results from direct detection experiments. In this paper, we extend our approach, using previously published data collected within low-background measurements with $\mathrm{Hf}$ and Os metal samples, along with the limits derived from bolometric measurements using $\mathrm{CaWO}_{4}$ and $\mathrm{PbWO}_{4}$ scintillating crystals. Although the NE bounds that we find are not as strong as the NR bounds that we derive from bolometer measurements, we will identify a path to leading constraints on WIMPy inelastic dark matter using the nuclear properties of target materials.

This paper is organized as follows. In Sec. II, we present the kinematics of inelastic dark matter scattering, including dark-matter-induced nuclear transitions and their nuclear response. A number of experiments are listed in Sec. III, and we show the resulting bounds on inelastic dark matter in Sec. IV. Finally, we conclude in Sec. V. 
TABLE I. Nuclear transitions from ground states (gs) to excited states (es) with energy level $\Delta E$ for Hf, Os, and Hg isotopes along with their spin, parity, and reduced transition probability information. The detection efficiencies $\eta$ near the transition energies are also listed. The most constraining isotopes and transitions are listed with the abundances (see Appendix A for a more complete list of transitions and bounds). The gamma background for nuclear excitation (NE) near specific deexcitation energies to the ground states is given at $68 \%$ C.L. in the last column. See Sec. III B for details.

\begin{tabular}{lccccccc}
\hline \hline Isotope & Abundance $(\%)$ & $J_{\mathrm{gs}}^{p}$ & $J_{\mathrm{es}}^{p}$ & $\Delta E(\mathrm{keV})$ & $B(E 2)($ W.u.) & $\eta(\%)$ & $\mathrm{Bkg}(\mathrm{mBq} / \mathrm{kg})$ \\
\hline${ }^{177} \mathrm{Hf}$ & 18.60 & $7 / 2^{-}$ & $9 / 2^{-}$ & 112.9500 & $282(8)[68]$ & 9.64 & 0.9 \\
${ }^{178} \mathrm{Hf}$ & 27.28 & $0^{+}$ & $2^{+}$ & 93.1803 & $160(3)[69]$ & 7.37 & 2.2 \\
${ }^{180} \mathrm{Hf}$ & 35.08 & $0^{+}$ & $2^{+}$ & 93.3240 & $154.8(21)[70]$ & 7.37 & 2.2 \\
${ }^{189} \mathrm{Os}$ & 16.15 & $3 / 2^{-}$ & $1 / 2^{-}$ & 36.17 & $27(7)[71]$ & 0.695 & 0.40 (projection) \\
& & $3 / 2^{-}$ & $5 / 2^{-}$ & 69.54 & $100(10)[71]$ & 1.75 & 0.16 \\
${ }^{201} \mathrm{Hg}$ & 13.17 & $3 / 2^{-}$ & $1 / 2^{-}$ & 1.5648 & $\sim 34[72]$ & 50 & 0.0056 (projection) \\
\hline \hline
\end{tabular}

\section{KINEMATICS OF INELASTIC DARK MATTER SCATTERING}

In models of inelastic DM, the dark sector comprises two states $\chi_{1}$ and $\chi_{2}$ with a mass splitting $\delta=M_{\chi_{2}}-M_{\chi_{1}}$. At tree level, $\chi_{2}$ couples to $\chi_{1}$ via a scalar or a gauge boson field, but the self-coupling $\bar{\chi}_{1} \chi_{1}$ or $\bar{\chi}_{2} \chi_{2}$ is loopsuppressed. In the simplest case, we consider the Lagrangian $\mathcal{L} \supset g \phi \bar{\chi}_{2} \chi_{1}+$ H.c. Since $\chi_{2}$ is heavier than $\chi_{1}$, after freeze-out we expect $\chi_{2}$ to be depleted either though decay or through the interconversion process $\chi_{2} \chi_{2} \rightarrow \chi_{1} \chi_{1}$ when the temperature of the Universe falls below the mass splitting, $T<\delta[44,73]$. As a consequence, the dark matter today is dominated by $\chi_{1}$.

We consider two types of dark matter interactions in the detector. The first is nuclear recoil (NR), where the target nucleus displaces, and $\chi_{1}$ transforms to $\chi_{2}$. In addition, $\chi_{1}$ may also interact with the valence nucleons and instigate nuclear excitation (NE) into an unstable state $N^{*}$. As long as the mediator mass $M_{\phi}$ is much larger than the momentum transfer $q$, the interaction can be described by the effective Lagrangian

$$
\mathcal{L} \sim \frac{1}{\Lambda^{2}} \bar{\chi}_{2} \chi_{1} \bar{N}^{*} N
$$

where the UV scale $\Lambda$ is set by the mediator mass and the coupling between the dark sector and the SM. We define the quantity $\Delta$, which represents the sum of the DM mass splitting $\delta$ and the excitation energy $\Delta E \equiv E_{N^{*}}-E_{N}$ :

$$
\Delta \equiv \delta+\Delta E
$$

$\Delta E$ vanishes in NR-only scattering. The kinematics of both interactions follows:

$$
\frac{\vec{q}_{i}^{2}}{2 M_{\chi_{1}}}=\frac{\vec{q}_{f}^{2}}{2 M_{\chi_{2}}}+\frac{\vec{q}^{2}}{2 M_{N}}+\Delta,
$$

where the momentum transfer $\vec{q}=\vec{q}_{f}-\vec{q}_{i}$. Equation (3) can be written

$$
\frac{q^{2}}{2 \mu_{\chi N}}-q v \cos \theta+\Delta=0
$$

where $\mu_{\chi N} \equiv M_{\chi} M_{N} /\left(M_{\chi}+M_{N}\right)$ is the reduced mass of the DM-nucleus system and $\theta$ is the laboratory frame scattering angle. We also define $M_{\chi} \equiv M_{\chi_{1}} \simeq M_{\chi_{2}}$, which is valid so long as $M_{\chi} \gg \delta$. We will assume the dark matter velocity $v$ follows a Maxwellian distribution with $v_{0}=220 \mathrm{~km} / \mathrm{s}$ truncated at the escape velocity $v_{\text {esc }}=600 \mathrm{~km} / \mathrm{s}$ in the Earth frame, assuming Earth's velocity is $v_{e}=240 \mathrm{~km} / \mathrm{s}$ [74]. We refer the reader to Refs. [74-77] for detailed discussions of uncertainties on the halo parameter values. The minimum and maximum momentum transfer is obtained at $|\cos \theta|=1$, which gives

$$
q_{\min / \max }=\mu_{\chi N} v\left[1 \mp \sqrt{1-2 \frac{\Delta}{\mu_{\chi N} v^{2}}}\right],
$$

and the minimum and maximum recoil energy is simply obtained via the relationship $E_{R}=q^{2} /\left(2 M_{N}\right)$. The minimum dark matter velocity required to scatter off a nucleus with any recoil energy is, thus,

$$
v_{\min }=\sqrt{2 \frac{\Delta}{\mu_{\chi N}}}
$$

Larger dark matter kinetic energy is, therefore, required for inelastic scattering as $\Delta$ increases. Consequently, the maximum mass splitting that can be probed in an experiment is

$$
\delta_{\max }=\frac{1}{2} \mu_{\chi N}\left(v_{e}+v_{\mathrm{esc}}\right)^{2}-\Delta E,
$$

which grows for larger reduced mass $\mu_{\chi N}$ and, hence, heavier target nuclei. For a specific nuclear recoil energy, the minimum dark matter velocity for an interaction to occur can also be obtained from Eq. (4): 


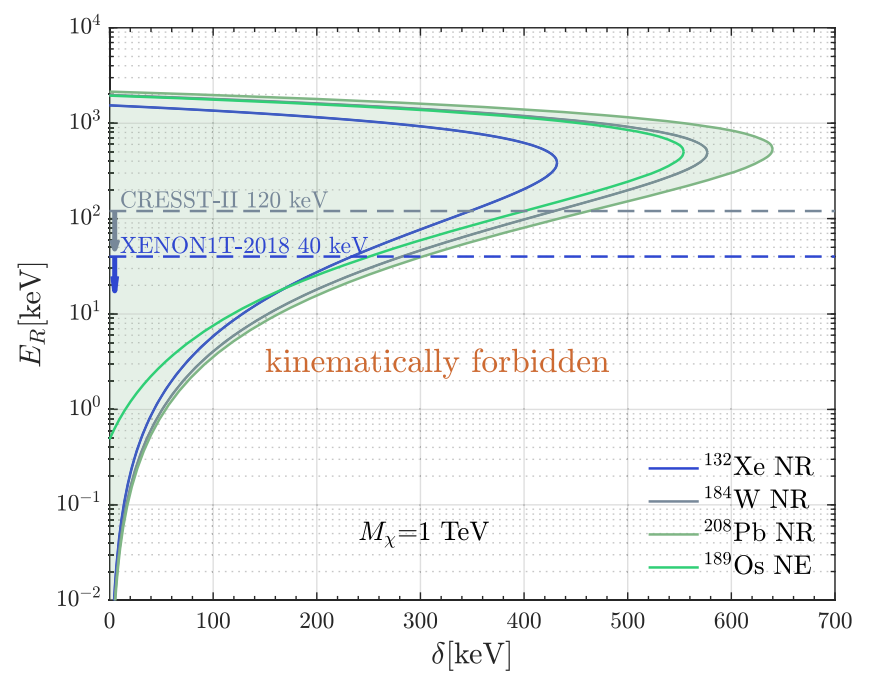

FIG. 2. Kinematics of inelastic dark matter with mass splitting $\delta$ scattering with a target nucleus with and without nuclear transition. Kinematically allowed recoil energies are shown for ${ }^{132} \mathrm{Xe},{ }^{184} \mathrm{~W},{ }^{208} \mathrm{~Pb}$, and ${ }^{189} \mathrm{Os}$ with the solid lines. The region for ${ }^{208} \mathrm{~Pb}$ is further shaded. We consider NR for the first three target nuclei and $\mathrm{NE}$ to the $36.17 \mathrm{keV}$ excited state for ${ }^{189} \mathrm{Os}$. The maximum recoil energy analyzed in XENON1T 2018 is about $40 \mathrm{keV}$ and that of CRESST-II [79] is about $120 \mathrm{keV}$, as depicted by the horizontal dashed lines. These limit the experiments' respective sensitivities to higher mass splittings. In this figure, the dark matter mass is fixed at $M_{\chi_{1}} \simeq M_{\chi_{2}}=1 \mathrm{TeV}$.

$$
v_{\min }\left(E_{R}\right)=\frac{1}{\sqrt{2 M_{N} E_{R}}}\left(\frac{M_{N}}{\mu_{\chi N}} E_{R}+\Delta\right) .
$$

The kinematics of NR scattering is shown in Fig. 2 for a few nuclei. For each isotope, the region to the left of the corresponding solid curve represents allowed values of $E_{R}$, given the IDM mass splitting $\delta$. The shape of these curves is determined by the DM velocity distribution. Focusing first on xenon experiments (solid green line), the limitation is twofold: (i) the $\mathrm{Xe}$ atomic mass limits the maximum transfer as in Eq. (7). (ii) The analyzed recoil energy, shown here for XENON1T as a dashed blue line, is restricted in most xenon experiments. For the recoil energy range of $1-40 \mathrm{keV}$, the maximum mass splitting that can be constrained is, thus, around $250 \mathrm{keV}$. Recently, XENON1T reported the event spectrum up to the electron recoil energies of $210 \mathrm{keV}$ [43]. A dedicated analysis of nuclear recoils at high energy would be required to constrain mass splitting above $200 \mathrm{keV}$, but the sensitivity will still be limited to $\delta<450 \mathrm{keV}$ due to the limited mass number of $\mathrm{Xe}$ isotopes. Conversely, tungsten (solid gray line) is a heavy and stable element, well suited to exploring high mass splittings. CRESST-II $\left(\mathrm{CaWO}_{4}\right.$, dashed gray line) analyzed recoil data up to $120 \mathrm{keV}$, which translates to a mass splitting of about $420 \mathrm{keV}$. Although the CRESST-III [78] data are also available, the detector was optimized to sub-GeV mass dark matter detection with better performance at low recoil energies. Therefore, we still expect CRESST-II to set the leading bound at higher mass splittings.

In addition to NR interactions in traditional direct detection experiments, we are also interested in deexcitation gamma quanta in NE scattering: $\chi_{1}+N \rightarrow \chi_{2}+$ $N^{*} \rightarrow \chi_{2}+N+\gamma$, whose kinematics is also illustrated in Fig. 2 for Os. Depending on the IDM model, $\chi_{2}$ may also decay to SM or invisible particles. If $\chi_{2}$ decays to photon(s) promptly inside the detector, the decay photon could be resolved as a signature of inelastic scattering; otherwise, $\chi_{2}$ may leave the detector. We conservatively assume the latter. For both NR and NE, the scattering rate is given by

$$
R=\sum_{i} N_{T_{i}} \frac{\rho_{\chi}}{M_{\chi}} \int_{v_{\min }}^{v_{\max }} d v v f(v) \int_{E_{R, \min }}^{E_{R, \max }} \frac{d \sigma_{\chi N}}{d E_{R}} d E_{R},
$$

where $N_{T_{i}}$ is the number of the target nuclei of a given isotope in the detector, $\rho_{\chi}=0.4 \mathrm{GeV} \mathrm{cm}^{-3}$ is the local dark matter density, and $f(v)$ is the one-dimensional Maxwellian velocity distribution. The minimum and maximum recoil energies can be found from Eq. (5). The differential cross section is

$$
\frac{d \sigma_{\chi N}}{d E_{R}}=\frac{\sigma_{n} M_{N}}{2 v^{2} \mu_{\chi n}^{2}} S\left(E_{R}\right)
$$

where $\sigma_{n}$ is the per-nucleon scattering cross section and the nuclear response is encapsulated in $S\left(E_{R}\right)$. For NR, this is just the Helm form factor. In the case of NE, dark matter interacts only with the few valence nucleons on the surface shell of the nucleus, where the nuclear response depends on the momentum transfer without the $A^{2}$ enhancement factor in $S\left(E_{R}\right)$. As first derived in Ref. [80], the nuclear response function is written as

$$
S(\vec{q})=\sum_{L}\left|\left\langle J_{f}|| j_{L}(q r) Y_{L M}(\hat{r})|| J_{i}\right\rangle\right|^{2},
$$

where the sum runs over all allowed even angular momentum states within the range $\left|J_{i}-J_{f}\right| \leq L \leq\left|J_{i}+J_{f}\right|$. Here, $J_{i}$ and $J_{f}$ are, respectively, the initial and final angular momenta, $j_{L}$ are spherical Bessel functions, and $Y_{L M}$ are spherical harmonics. The calculation of the response function is usually quite involved for heavy nuclei due to the complexity of the valence structure and the deformation of the nucleus. Fortunately, we may use the reduced transition probability in the nuclear E2 transition to approximately eliminate the angular matrix element, which is defined as

$$
B(E 2)=\frac{1}{2 J_{i}+1}\left|\left\langle J_{f} \| e r^{2} Y_{2}|| J_{i}\right\rangle\right|^{2},
$$

with $Y_{2}$ the spherical tensor operator. Assuming the nuclear transition probability density is peaked near the surface of the nucleus, we find [81] 


$$
S(\vec{q})=\frac{A^{2}}{Z^{2}}\left(2 J_{i}+1\right) j_{2}(q R)^{2} \frac{B(E 2)}{e^{2} R^{4}},
$$

with the nucleus radius $R \simeq 1.2 \times A^{1 / 3} \mathrm{fm}$. The measured reduced transition probabilities $B(E 2)$ are listed in Table I. These are expressed in terms of the Weisskopf unit (W.u.) [82] defined as [83]

$$
B_{\mathrm{W} . u .}(E \lambda)=\frac{1.2^{2 \lambda}}{4 \pi}\left(\frac{3}{\lambda+3}\right)^{2} A^{2 \lambda / 3} e^{2} \mathrm{fm}^{2 \lambda},
$$

for a general $E \lambda$ transition. Since higher multipole transitions involving $j_{L}^{2}$ are strongly suppressed, we consider only the leading contribution to the nuclear response where $L=2$.

\section{EXPERIMENTS}

Apart from a few notable exceptions, there have been no dedicated experiments searching for DM-nucleus interactions in heavy elements $Z \gtrsim 54$. However, these isotopes have been considered in many calibration experiments as well as searches for rare nuclear decays with the objective of better understanding their nuclear properties. Here, we list a number of promising target compounds, along with published data. We separate these out into two categories: $\mathrm{NR}$, leading to nuclear recoils identifiable in scintillating bolometers, and NE, using gamma-ray detectors.

Our strategy is to identify events which could have resulted from a DM-nucleus interaction and translate the corresponding rate into a limit on the DM interaction rate. This is a conservative approach which, in the absence of a detailed background model for each experiment, can be used only for limit setting rather than discovery. We will recast these constraints on the rates into constraints on IDM model parameters in Sec. IV.

\section{A. Nuclear recoil measurements}

\section{1. $\mathrm{CaWO}_{4}$}

Crystals used by the CRESST (II-III) [84,85] experiments contain a sizable fraction of tungsten and are, thus, a good target for IDM searches. However, the analysis region for the CRESST DM runs was limited to low recoil energies, so we turn to the results of the crystal characterization runs. The radiopurity of a variety of $\mathrm{CaWO}_{4}$ crystals was investigated at the Gran Sasso National Laboratory (LNGS). We employ measurements between 2009 and 2011 with the CRESST main setup where the scintillation light and phonon signals were both collected. The radiopurity results for a single crystal named Daisy are shown in Fig. 2 of Ref. [60], representing $90.10 \mathrm{~kg}$ - days of exposure. The two-channel readout (scintillation and phonon) facilitates background event discrimination. The phonon channel was calibrated with $\alpha$ particles, which typically produce a light yield that is around $22 \%$ of the $\beta / \gamma$ light yield in $\mathrm{CaWO}_{4}$ crystals. Even less light is expected for nuclear recoils: For example, the light yield for nuclear recoils from a neutron calibration run of CRESST using $\mathrm{CaWO}_{4}$ was found to be below $11 \%$ [86]. Conservatively, we will set bounds by counting all events observed in Daisy below the $\alpha$ band as dark matter candidates. Although the nuclear recoil energy is not available due to the lack of dedicated neutron calibration, we approximate it by the equivalent $\alpha$ energy up to an uncertainty of about $20 \%$, which does not impact our results in a notable way. With this criterion, we identify three dark matter event candidates between $300 \mathrm{keV}$ and $2 \mathrm{MeV}$, which yield an upper limit of 6.7 events at $90 \%$ C.L. Since the bolometric detector is sensitive to the total energy deposition in the heat channel, the efficiency is close to 1 in the energy range we consider.

\section{2. $\mathrm{PbWO}_{4}$}

Similarly, the $\alpha$ decay of lead isotopes was studied with $11.09 \mathrm{~kg} \cdot$ days of $\mathrm{PbWO}_{4}$ background measurements, by using the crystal as a scintillating bolometer at the LNGS [61]. A lower light yield is expected from crystals of this type, which for $\alpha$ particles can be estimated via [61]

$$
\begin{aligned}
\frac{\mathrm{LY}_{\alpha}}{(\mathrm{keV} / \mathrm{MeV})}= & (0.28 \pm 0.01)+(2.93 \pm 0.14) \times 10^{-5} \\
& \cdot\left(\frac{E_{\alpha}}{\mathrm{keV}}\right)
\end{aligned}
$$

where $E_{\alpha}$ is the $\alpha$ particle energy. Taking the events with a lower light yield than expected from $\alpha$ particles as dark matter candidates, we identify 12 events between $600 \mathrm{keV}$ and $2 \mathrm{MeV}$, which corresponds to an upper limit of 17.8 events at $90 \%$ C.L. Likewise, we approximate the nuclear recoil energy by the $\alpha$-equivalent energy, since most energy is deposited in the heat channel in both cases. The detection efficiency is also close to 1 . The results of $\mathrm{CaWO}_{4}$ and $\mathrm{PbWO}_{4}$ experiments are summarized in Table II.

TABLE II. Summary of results from $\mathrm{CaWO}_{4}$ and $\mathrm{PbWO}_{4}$ scintillating bolometer experiments, where the experimental exposure, analyzed energy window, and the number of dark matter candidates are listed. See Appendix B for details on DM event selection.

\begin{tabular}{lccc}
\hline \hline & $\begin{array}{c}\text { Exposure } \\
(\mathrm{kg} \cdot \text { day })\end{array}$ & $\begin{array}{c}\text { Phonon } \\
E(\mathrm{keV})\end{array}$ & $\begin{array}{c}\text { DM } \\
\text { events }\end{array}$ \\
\hline $\mathrm{CaWO}_{4}[60]$ & 90.10 & $300-2000$ & 3 \\
$\mathrm{PbWO}_{4}[61]$ & 11.09 & $600-2000$ & 12 \\
\hline \hline
\end{tabular}




\section{B. Nuclear excitation measurements}

\section{Os}

Recently, the search for rare alpha decay of osmium isotopes was conducted at the LNGS [87]. 117.96 grams of high-purity Os metal were cut into thin slices to reduce gamma absorption inside the metal. The slices were installed on a broad-energy germanium detector with high sensitivity and ultralow background. The accumulated energy spectrum was reported, with an exposure of $15851 \mathrm{~h}$. The relevant transition levels and the corresponding background rates of Os isotopes are summarized in Table I. The deexcitation gamma quanta will appear as a peak in the continuous spectrum. Nonobservation of the peak implies that the $68 \%$ C.L. upper limit on the transition rate (marked as "Bkg") can be estimated as

$$
R_{\mathrm{Bkg}}=\sqrt{s \cdot \sigma_{\epsilon}} /\left(I_{\gamma} \cdot \eta \cdot M \cdot t_{\mathrm{exp}}\right),
$$

where $s$ is the accumulated counts per $\mathrm{keV}$ as indicated by the measured energy spectrum. The $90 \%$ C.L. limit can be estimated by assuming Gaussian distribution, where the transition rate is multiplied by factor of 1.645 . The energy resolution or full width at half maximum (FWHM) is approximated by [87]

$$
\left(\frac{\sigma_{\epsilon}}{\mathrm{keV}}\right)=0.57(5)+0.029(2) \times \sqrt{E_{\gamma} / \mathrm{keV}},
$$

where $E_{\gamma}$ is the transition energy. $M$ and $t_{\text {exp }}$ are given by the mass of an Os isotope and the exposure time, respectively. The efficiency of the HPGe detector depends on details of the sample including geometry, density and chemical composition, and the energy of gamma quanta. In the absence of the original experimental data, we construct a polynomial fit to the efficiency curve as a function of $\ln \left(E_{\gamma}\right)$ [88] between 46.5 and $200 \mathrm{keV}$ and take the efficiency to be constant below $46.5 \mathrm{keV}$. ${ }^{1}$ A dedicated analysis may yield an $\mathcal{O}(1)$ correction to the efficiency adopted here. If intermediate states exist between the excited nucleus and its ground state, deexcitation may take place through the emission of gamma cascades. Picking a specific gamma energy for background analysis entails the branching ratio $I_{\gamma}$, which is $100 \%$ for all transitions listed in Table I. We, therefore, search for the deexcitation gamma and set bounds on the cross section by requiring that the dark matter scattering rate in Eq. (9) not exceed the measured transition rate.

\footnotetext{
${ }^{1}$ Direct communications with the authors of Ref. [87] show that the $36 \mathrm{keV}$ gamma is below the detection threshold, which we label as "projection" in Table I.
}

\section{2. $\mathrm{Hf}$}

Rare decay of hafnium isotopes were also investigated at the LNGS by measuring the internal background of $55.38 \mathrm{~g}$ of Hf foil using a modified HPGe detector [67]. Lowbackground data have been collected over 310.6 days. The most constraining transitions and the background rates are also listed in Table I. The corresponding limits on inelastic dark matter have been derived in Ref. [67].

\section{3. $\mathrm{Hg}$}

${ }^{201} \mathrm{Hg}$ is an ideal target NE gamma search because of its low-lying excited state at $1.6 \mathrm{keV}$. Since $\mathrm{Hg}$ is toxic, it is preferable to use mercury-containing compounds as the target for dark matter scattering. One such example is to use mercury cadmium telluride $\left(\mathrm{Hg}_{1-x} \mathrm{Cd}_{x} \mathrm{Te}\right)$. While this would suffer from a high contamination from ${ }^{113} \mathrm{Cd}$ beta decays, which will dominate over other background below $316 \mathrm{keV}$, increasing the fraction of $\mathrm{Hg}$ in the crystal would somewhat diminish this background component. Conservatively, we consider $x=0.2$. The ${ }^{113} \mathrm{Cd}$ decay background at $1.6 \mathrm{keV}$ can be obtained by rescaling the experimental data at $100 \mathrm{keV}$ according to the predicted decay spectrum in Fig. 5 of Ref. [89]. This $1.6 \mathrm{keV}$ gamma can be measured with an external low-threshold gamma detector such as a Ge cryogenic bolometer [90-92], which is to be installed in close contact with the $\mathrm{Hg}_{0.8} \mathrm{Cd}_{0.2} \mathrm{Te}$ target. The energy resolution of the Ge gamma detector is estimated to be $50 \mathrm{eV}$ around $1.6 \mathrm{keV}$ [92]. Assuming $10 \mathrm{~kg} \cdot \mathrm{yr}$ of $\mathrm{Hg}_{0.8} \mathrm{Cd}_{0.2} \mathrm{Te}$ exposure and $\sim 50 \%$ efficiency for gamma detection, we estimate the background rate to be $0.0004 \mathrm{mBq}$ per $\mathrm{kg}$ of $\mathrm{Hg}_{0.8} \mathrm{Cd}_{0.2} \mathrm{Te}$ (or, equivalently, $0.0056 \mathrm{mBq}$ per $\mathrm{kg}$ of ${ }^{201} \mathrm{Hg}$ ) at $1.6 \mathrm{keV}$ transition energy.

Although the DM analysis in this work is based on the measurement of the $1.6 \mathrm{keV}$ gamma in DM-induced NE, the DM limit can be further improved by investigating nuclear recoil signals. Since $\mathrm{Hg}_{0.8} \mathrm{Cd}_{0.2} \mathrm{Te}$ as a semiconductor is also sensitive to energy deposition, we can improve this setup by measuring the recoil energy of $\mathrm{Hg}$ with $\mathrm{Hg}_{0.8} \mathrm{Cd}_{0.2} \mathrm{Te}$. The coincident detection of nuclear recoil at $\mathrm{Hg}_{0.8} \mathrm{Cd}_{0.2} \mathrm{Te}$ and $1.6 \mathrm{keV}$ gamma at the $\mathrm{Ge}$ bolometer as DM signals provides a powerful way for background rejection. The exploration of this method is left for further work.

\section{RESULTS}

The constraints on dark matter-nucleon scattering based on the experiments described above are shown in Fig. 3, where we have set the DM mass to $M_{\chi}=1 \mathrm{TeV}$. For dark matter much heavier than the target nuclei, the cross section bound scales linearly with $M_{\chi}$.

As an illustration of IDM-induced nuclear transition, we show the bounds from the NE to different Os excited states in Fig. $3 .{ }^{189} \mathrm{Os}$ sets the leading bound for mass splittings below $520 \mathrm{keV}$, due to the collisional excitation to the 


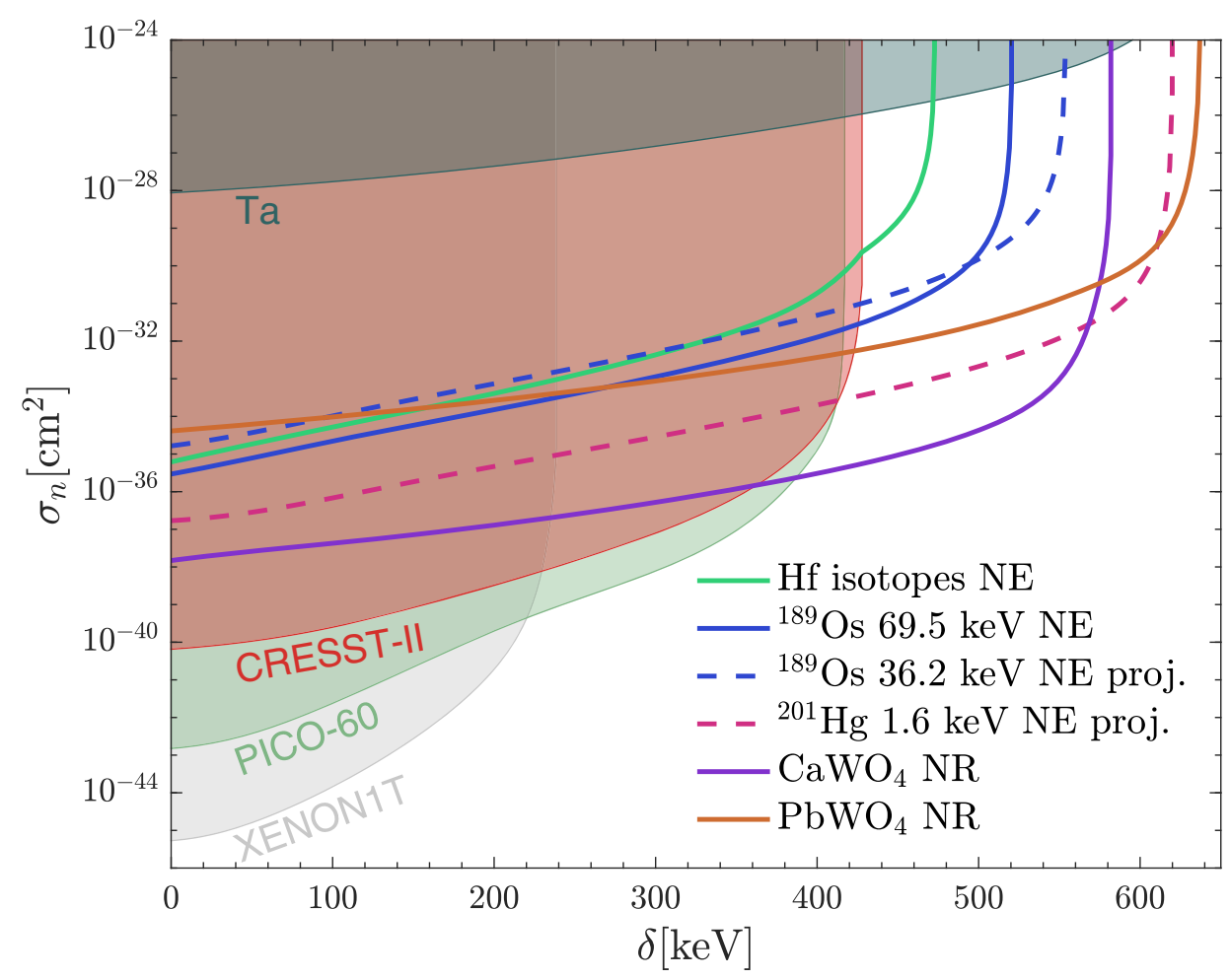

FIG. 3. Constraints on inelastic dark matter-nucleon scattering cross section at $90 \%$ C.L. We assume a dark matter mass $M_{\chi}=1 \mathrm{TeV}$, local dark matter density $\rho_{\chi}=0.4 \mathrm{GeV} / \mathrm{cm}^{3}$, and a Maxwellian distribution with the Earth velocity $v_{e}=240 \mathrm{~km} / \mathrm{s}$ and the escape velocity $v_{\text {esc }}=600 \mathrm{~km} / \mathrm{s}$. Limits based on data from PICO-60 [93], CRESST-II [79], XENON1T [94], and the ${ }^{180}$ Ta experiment [63] have been rederived accordingly and are shown with the shaded regions. The green, blue, and magenta lines show the cross section limits derived from inelastic dark matter scattering which induces nuclear transitions of $\mathrm{Hf}$ [67], Os [87], and $\mathrm{Hg}$, respectively, and the deexcitation gamma rays. Dashed lines are for future projections. The solid purple and orange lines depict the limits on inelastic dark matter from the recoil of heavy nuclei, derived in this work using data from $\mathrm{CaWO}_{4}[60]$ and $\mathrm{PbWO}_{4}[61]$ crystals, respectively (see the text for details).

$9.54 \mathrm{keV}$ state. Stronger bounds could be obtained from 505 to $553 \mathrm{keV}$ by considering the future measurement of $36.17 \mathrm{keV}$ transition gamma. We also set limits on inelastic dark matter by employing $\mathrm{Hf}$ rare decay measurements in a similar approach [67], which yields less stringent bounds compared with osmium owing to relatively higher transition energies and less-massive nuclei. We also propose to detect inelastic dark matter by investigating the deexcitation gamma yield of a mercury-containing compound. With $10 \mathrm{~kg} \cdot \mathrm{yr}$ of $\mathrm{HgCdTe}$ exposure as described above, ${ }^{201} \mathrm{Hg}$ is expected to yield leading limits among nuclear excitation IDM searches for mass splittings between 415 and $620 \mathrm{keV}$.

We have also derived the bounds for IDM NR scattering on $\mathrm{CaWO}_{4}$ and $\mathrm{PbWO}_{4}$ target crystals acting as scintillating bolometers. Thanks to the simultaneous light and phonon detection, nuclear recoils can be distinguished on a statistical basis from electron recoil and gamma background. These bounds are more stringent than those from $\mathrm{Hf}$ and $\mathrm{Os}$ isotopes for all mass splittings. In particular, the $\mathrm{CaWO}_{4}$ experiment dominates the 386$573 \mathrm{keV}$ range, and the $\mathrm{PbWO}_{4}$ measurements constrain the mass splitting onward up to $636 \mathrm{keV}$. Nevertheless, ${ }^{201} \mathrm{Hg}$ is still expected to lead the 570 and $604 \mathrm{keV}$ mass splitting.
We also show the constraints from XENON1T, PICO-60 [93], CRESST-II [79], and ${ }^{180 m} \mathrm{Ta}$ [63]. The XENON Collaboration reported the results for WIMP searches from about 1 tonne $\cdot \mathrm{yr}$ of data collection [94] in 2018. We reproduce the results following the efficiency and event selection in the Xenon1T-2018 code [95]. Two events were observed with 0.9 tonne reference mass and 278.8 days live time, and the background is expected to be $1.62 \pm 0.28$. This translates to an upper limit of 3.7 events at $90 \%$ C.L. Discrimination of nuclear recoil, electron recoil, and other backgrounds on an event-by-event basis requires full likelihood analysis, which is beyond the scope of this work. We derive the bound on inelastic dark matter by integrating over the nuclear recoil energy between 0 and $40.9 \mathrm{keV}$, with the efficiencies taking care of the low-energy cut. The PICO-60 [93] and CRESST-II [79] bounds in Ref. [96] are recomputed with the dark matter velocity distribution assumed in this paper, ${ }^{2}$ and the Ta bound [63] is reproduced with the same assumptions.

We extend the bound to lower and higher dark matter masses in Fig. 4. The colors depict the smallest cross

\footnotetext{
${ }^{2}$ As in Ref. [96], we consider the recoil energy up to $1 \mathrm{MeV}$ for PICO and the 30-120 keV recoil energy for CRESST-II.
} 


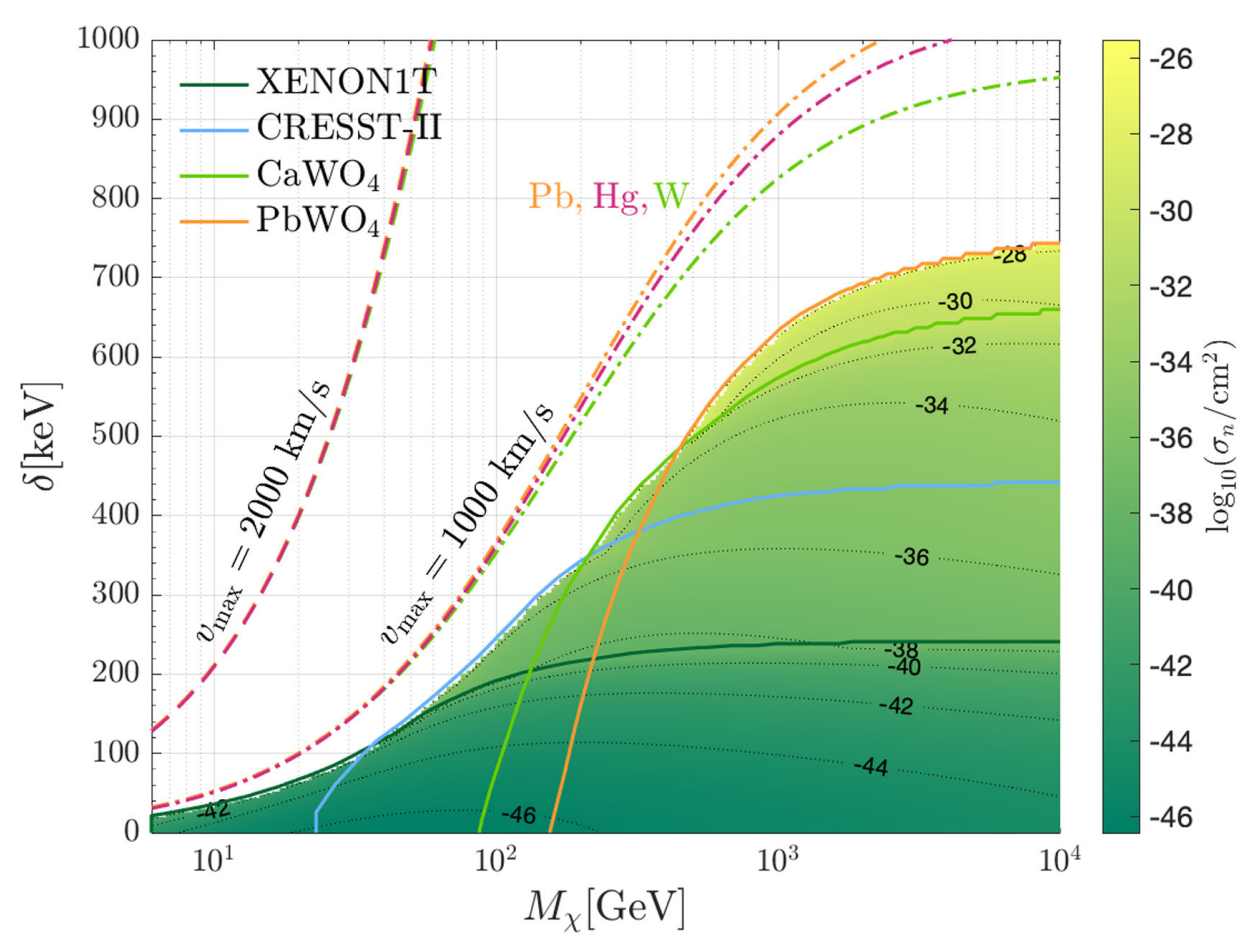

FIG. 4. Constraints on inelastic dark matter-nucleon scattering for different dark matter masses and mass splittings. The colored regions to the right of the solid lines are excluded by existing experiments, including XENON1T [94], CRESST-II [79], and the bolometric searches with $\mathrm{CaWO}_{4}[60]$ and $\mathrm{PbWO}_{4}[61]$ scintillating crystals. Colors and contour lines mark the minimum cross section at $90 \%$ C.L. that can be excluded by the combination of experiments. The white region on the upper left is not kinematically accessible in existing experiments. Each experiment is sensitive to a limited amount of parameter space as enclosed by the solid lines. If a subcomponent of the dark matter is boosted to higher velocities, more parameter space can be excluded. The dash-dotted lines show the projected reach for maximum dark matter velocities of $v_{\max }=1000 \mathrm{~km} / \mathrm{s}$, and the dashed lines for $v_{\max }=2000 \mathrm{~km} / \mathrm{s}$. The green, orange, and red broken lines, respectively, correspond to future dark matter search through scattering with tungsten (nuclear recoil), lead (nuclear recoil), and mercury (nuclear excitation), regardless of the detection threshold.

sections that are excluded with the combination of experiments listed above. The mass splitting that can be constrained rises as dark matter mass increases. The white region is not kinematically accessible due to the limitation of $\mu_{\chi N}$ and dark matter velocity, as is evident from Eq. (7). For $\delta \lesssim 200 \mathrm{keV}$, leading bounds are set by XENON1T. In the intermediate regime, the $\mathrm{CaWO}_{4}$ experiment remains the most sensitive search, which is overtaken by $\mathrm{PbWO}_{4}$ at high mass splittings. Current limits from dark-matterinduced nuclear excitations remain subdominant.

Apart from the target nucleus mass, the maximum DM velocity is a limiting factor for probing high mass splittings. Although DM is considered to be virialized, many recent works have shown that a subdominant component could be boosted to high velocity, through cosmic-ray (e.g., [97-100]) or neutrino (e.g., [101-103]) scattering or stellar acceleration (e.g., [104]), annihilation (e.g., [105,106]), semiannihilation (e.g., $[107,108]$ ), or decay of heavy states (e.g., $[109,110])$. Cosmic-ray upscattered inelastic dark matter with a vector mediator has recently been studied in Ref. [111]. Regardless of boost mechanism, we illustrate the kinematic reach of future heavy nuclei experiments to boosted IDM particles in Fig. 4, for DM velocities of 1000 (dot-dashed lines) and $2000 \mathrm{~km} / \mathrm{s}$ (dashed lines) assuming $\mathrm{W}, \mathrm{Pb}(\mathrm{NR})$, and $\mathrm{Hg}$ (NE) nuclei as targets. The full parameter space in $M_{\chi}-\delta$ shown in Fig. 4 can largely be covered by if $v_{\max }=2000 \mathrm{~km} / \mathrm{s}$. We choose to cap the mass splitting at $\mathrm{MeV}$, beyond which the heavier state may decay to $e^{ \pm}$in the detector. An exploration of the prompt decay scenario is left for future work.

\section{CONCLUSIONS}

As direct detection experiments close in on the window of possible WIMP candidates in the $\mathrm{GeV}$ range, inelastic dark matter remains comparatively unconstrained by experiments with light nuclei, due to kinematical suppression, as can be seen from Eq. (7). We have extended the scope of this search by investigating nuclear recoil and nuclear excitation events in experiments with heavy nuclei using two distinct experimental approaches.

In the first scenario, target nuclei recoil due to the momentum transferred by dark matter scattering. In a scintillating bolometer, recoil energies up to about $2 \mathrm{MeV}$ will exhibit distinct features from electron or gamma background due to the low quenching in nuclear recoil. 
We reinterpret the high phonon energy events with low scintillation yield as dark matter scattering candidate events in experiments with $\mathrm{CaWO}_{4}$ and $\mathrm{PbWO}_{4}$ scintillating bolometers and set bounds on mass splitting up to $636 \mathrm{keV}$ for $\mathrm{TeV}$ dark matter. This is the largest splitting explored thus far in the literature. Our limits are conservative, as we do not have access to the raw data in the experiments. The discrimination of dark matter candidates from background on an event-byevent basis will strengthen the bounds on inelastic dark matter. The constraints can be further improved in the future with low-background scintillating crystals [112]. In particular, the RES-NOVA [113] experiment with large exposure and better background modeling using Monte Carlo may significantly improve the limits on inelastic dark matter if it is repurposed for dark matter search. We have also revisited the bounds from XENON1T, PICO-60, CRESST-II, and Ta. A likelihood analysis or the study of the high recoil energy data in XENON1T may notably extend the current xenon bound below $450 \mathrm{keV}$.

In the second scenario, target nuclei are excited to higher-energy states through inelastic collision with dark matter. In the absence of any background process that could yield such an excitation, the deexcitation gamma photons are unique signals of dark matter. In contrast to the difficult measurements with cryogenic scintillating bolometers calibrated at high recoil energies, the detection of nuclear excitation is simply viable with a HPGe detector, at the cost of excitation energy and the loss of coherent enhancement in the interaction cross section. We have shown that a recent Os deexcitation gamma search constrains the mass splitting up to $553 \mathrm{keV}$, and a future $\mathrm{Hg}$ experiment could extend the sensitivity up to $620 \mathrm{keV}$.
A key feature of WIMPy inelastic dark matter resides in its kinematics tightly coupled to the nuclear masses. As we are marching toward the end of the period table, heavy elements are exploited to tackle the high mass splitting regime, rapidly shrinking this large parameter space. Dedicated high-mass, high-energy deposition experiments are required to perfect this approach, in combination with more sophisticated analyses of the dark matter flux at different velocities.

\section{ACKNOWLEDGMENTS}

We thank Joseph Bramante and Peng Wang for helpful discussions and Harikrishnan Ramani for correspondence on Ta constraints [63]. We also thank the CRESST group at MPI Munich and Alexander Derbin for openly discussing their Tm data and fruitful collaboration and Stefano Pirro for communications on Ge detector. S. N., N. S., and A. C. V. are supported by the Arthur B. McDonald Canadian Astroparticle Physics Research Institute and NSERC, with equipment funded by the Canada Foundation for Innovation and the Province of Ontario, and housed at the Queen's Centre for Advanced Computing. Research at Perimeter Institute is supported by the Government of Canada through the Department of Innovation, Science, and Economic Development and by the Province of Ontario.

\section{APPENDIX A: BOUNDS FROM DARK-MATTER- INDUCED NUCLEAR EXCITATIONS}

We explore more isotopes in this appendix. Hf, Os, and $\mathrm{Hg}$ isotopes and their transitions studied in this work are listed in Table III. Bounds on IDM derived accordingly are

TABLE III. Nuclear transitions from ground states (gs) to excited states (es) with energy level $\Delta E$ for Hf, Os, and Hg isotopes along with their spin, parity, reduced transition probability information, and the isotope abundances. The deexcitation gamma energies $E_{\gamma}$ employed in the analysis are listed with their branching ratio $I_{\gamma}$ (normalized to 1). The gamma background for NE near specific deexcitation energies to the ground states is given at $68 \%$ C.L. in the last column. See Sec. III B for details.

\begin{tabular}{|c|c|c|c|c|c|c|c|c|}
\hline Isotope & Abundance (\%) & $J_{\mathrm{gs}}^{p}$ & $J_{\mathrm{es}}^{p}$ & $\Delta E(\mathrm{keV})$ & $B(E 2)$ (W.u.) & $E_{\gamma}(\mathrm{keV})$ & $I_{\gamma}(\%)$ & $\mathrm{Bkg}(\mathrm{mBq} / \mathrm{kg})$ \\
\hline${ }^{174} \mathrm{Hf}$ & 0.16 & $0^{+}$ & $2^{+}$ & 90.985 & 152(8) [114] & 91.00 & 100 & 3.8 \\
\hline${ }^{176} \mathrm{Hf}$ & 5.26 & $0^{+}$ & $2^{+}$ & 88.349 & 183(7) [115] & 88.34 & 100 & 3.1 \\
\hline${ }^{177} \mathrm{Hf}$ & 18.60 & $7 / 2^{-}$ & $9 / 2^{-}$ & 112.9500 & 282(8) [68] & 112.9498 & 100 & 0.9 \\
\hline${ }^{178} \mathrm{Hf}$ & 27.28 & $0^{+}$ & $2^{+}$ & 93.1803 & $160(3)$ [69] & 93.1803 & 100 & 2.2 \\
\hline${ }^{179} \mathrm{Hf}$ & 13.62 & $9 / 2^{+}$ & $11 / 2^{+}$ & 122.7904 & 245(14) [116] & 122.793 & 100 & 0.9 \\
\hline${ }^{180} \mathrm{Hf}$ & 35.08 & $0^{+}$ & $2^{+}$ & 93.3240 & $154.8(21)[70]$ & 93.324 & 100 & 2.2 \\
\hline \multirow[t]{2}{*}{${ }^{187} \mathrm{Os}$} & \multirow[b]{2}{*}{1.8794} & $1 / 2^{-}$ & $3 / 2^{-}$ & 74.356 & $50_{-50}^{+60}[117]$ & 74.30 & 100 & 0.25 \\
\hline & & $1 / 2^{-}$ & $5 / 2^{-}$ & 75.016 & $38(10)[117]$ & 64.31 & 54.3 & 0.31 \\
\hline \multirow[t]{3}{*}{${ }^{189} \mathrm{Os}$} & \multirow{3}{*}{16.152} & $3 / 2^{-}$ & $1 / 2^{-}$ & 36.17 & $27(7)[71]$ & 36.17 & 100 & 0.40 \\
\hline & & $3 / 2^{-}$ & $5 / 2^{-}$ & 69.54 & $100(10)[71]$ & 69.53 & 99.8 & 0.16 \\
\hline & & $3 / 2^{-}$ & $3 / 2^{-}$ & 95.27 & 14(3) [71] & 59.06 & 75.5 & 0.20 \\
\hline \multirow[t]{3}{*}{${ }^{201} \mathrm{Hg}$} & \multirow{3}{*}{13.17} & $3 / 2^{-}$ & $1 / 2^{-}$ & 1.5648 & $\sim 34[72]$ & 1.5648 & 100 & 0.0056 (projection) \\
\hline & & $3 / 2^{-}$ & $5 / 2^{-}$ & 26.272 & $2.4(8)[72]$ & 26.34 & 100 & 0.0060 (projection) \\
\hline & & $3 / 2^{-}$ & $3 / 2^{-}$ & 32.145 & $20(9)[72]$ & 32.19 & 50.5 & 0.012 (projection) \\
\hline
\end{tabular}




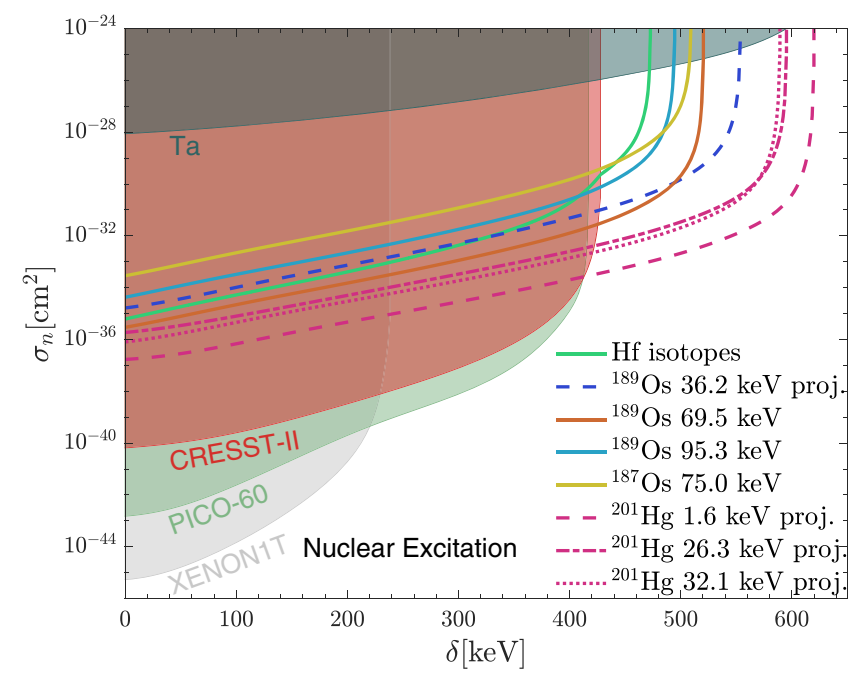

FIG. 5. Constraints on inelastic dark matter-nucleon scattering cross section derived from nuclear excitations at $90 \%$ C.L. On top of Fig. 3, we show in addition the existing bounds from the gamma measurements of the excitation of ${ }^{189} \mathrm{Os}$ to the $95.3 \mathrm{keV}$ state and ${ }^{187} \mathrm{Os}$ to the $75.0 \mathrm{keV}$ state [87]. Projection of nuclear

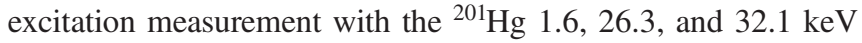
excited states and their deexcitations are depicted by dashed, dash-dotted, and dotted lines, respectively.

shown in Fig. 5. For constraints from individual $\mathrm{Hf}$ isotope, see [67]. We do not include ${ }^{187} \mathrm{Os} 74.4 \mathrm{keV}$ excitation in the analysis, since the measured $B(E 2)$ is subject to large uncertainty. The projected background rates of transitioning to ${ }^{201} \mathrm{Hg} 26.3$ and $32.1 \mathrm{keV}$ excited

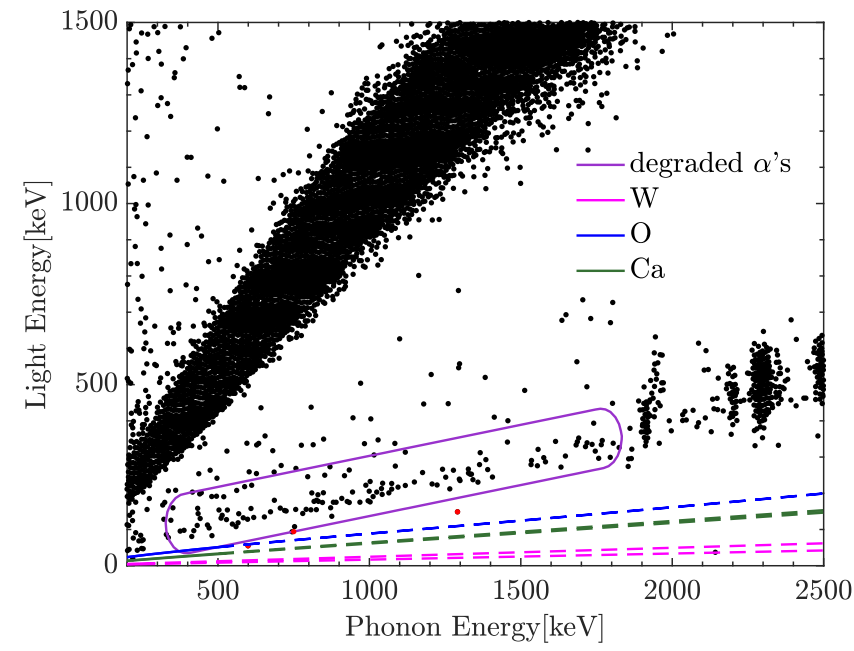

FIG. 6. Event discrimination in the $\mathrm{CaWO}_{4}$ scintillating bolometer experiment. The purple band corresponds to the degraded $\alpha$ events in Ref. [60]. The blue, green, and magenta lines show the measured light yields of $\mathrm{O}, \mathrm{Ca}$, and $\mathrm{W}$, respectively, in nuclear recoil at $90 \%$ C.L. [118]. The light yield of W recoil is measured up to $\sim 240 \mathrm{keV}$ recoil energy, and that of $\mathrm{O}$ and $\mathrm{Ca}$ recoils are measured up to $\sim 500 \mathrm{keV}$, beyond which we depict light yields as dashed lines. Events painted red below the purple $\alpha$ band are considered as dark matter candidates.
TABLE IV. $1 \sigma$ range of parameters for the light yields of $\mathrm{O}$ and Ca taken from Ref. [118].

\begin{tabular}{lccc}
\hline \hline & LY $^{\infty}$ & $f$ & $\lambda(\mathrm{keV})$ \\
\hline $\mathrm{O}$ & $0.07908 \pm 0.00002$ & $0.7088 \pm 0.0008$ & $567.1 \pm 0.9$ \\
$\mathrm{Ca}$ & $0.05949 \pm 0.00078$ & $0.1887 \pm 0.0022$ & $801.3 \pm 18.8$ \\
\hline \hline
\end{tabular}

states are derived similarly to the $1.6 \mathrm{keV}$ state by rescaling the ${ }^{113} \mathrm{Cd}$ decay data [89] and by assuming $50 \%$ efficiency.

We find the bounds from ${ }^{187} \mathrm{Os} 75.0 \mathrm{keV}$ and ${ }^{189} \mathrm{Os}$ $95.3 \mathrm{keV}$ transitions are inferior to the ${ }^{189} \mathrm{Os} 69.5 \mathrm{keV}$ constraints. Limits from ${ }^{201} \mathrm{Hg} 26.3$ and $32.1 \mathrm{keV}$ are also weaker than $1.6 \mathrm{keV}$ due to the kinematical suppression. Other elements that feature low transition energy and high mass include ${ }^{181} \mathrm{Ta},{ }^{183} \mathrm{~W},{ }^{193} \mathrm{Ir}$, and ${ }^{197} \mathrm{Au}$, which could be adopted as nuclear targets for future IDM searches.

\section{APPENDIX B: EVENT SELECTION FOR SCINTILLATING BOLOMETER EXPERIMENTS}

In Fig. 6, we show the dark matter candidate events in the $\mathrm{CaWO}_{4}$ scintillating bolometer experiment. The light yields (LYs) of $\mathrm{O}, \mathrm{Ca}$, and $\mathrm{W}$ in nuclear recoil have been studied in Ref. [118], which all approach a constant at high energies. The LYs of $\mathrm{O}$ and $\mathrm{Ca}$ can be parametrized as

$$
\operatorname{LY}\left(E_{R}\right)=\operatorname{LY}^{\infty}\left(1+f e^{-E_{R} / \lambda}\right)
$$

with $\mathrm{LY}^{\infty}$ being the LY at $E_{R}=\infty$. The relevant parameters are given in Table IV. The LY of W in nuclear recoil is measured to be $0.0208 \pm 0.0024$. The LYs are depicted as

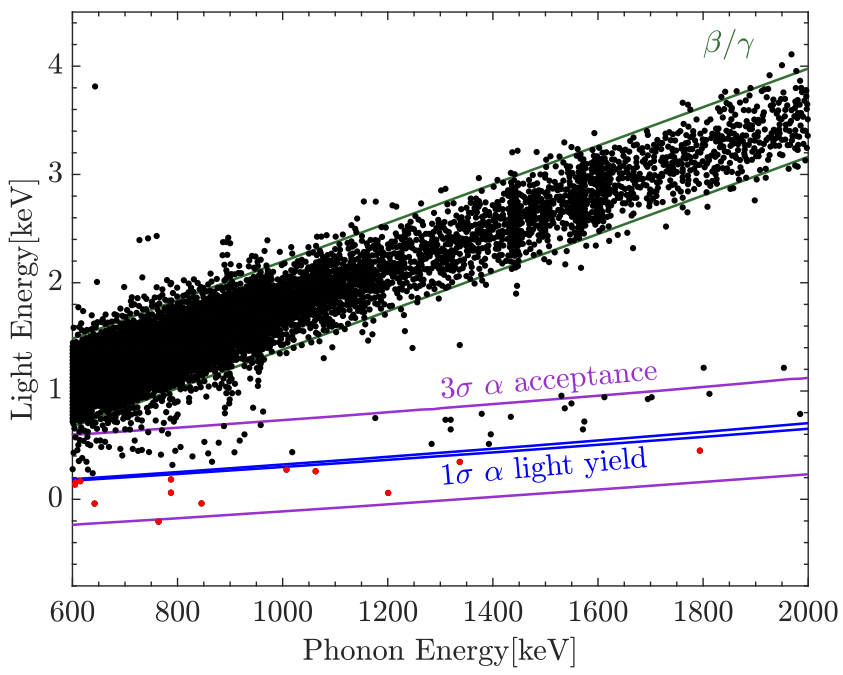

FIG. 7. Event discrimination in the $\mathrm{PbWO}_{4}$ scintillating bolometer experiment. The green and purple lines correspond to the $3 \sigma$ acceptance region for $\beta / \gamma$ and $\alpha$ events adapted from Ref. [61], respectively. The blue lines represent the $1 \sigma$ region of $\alpha$ events based on the light yield of $\alpha$ particles. Events painted red below the blue lines are considered as dark matter candidates. 
solid and dashed lines in Fig. 6 at 90\% C.L. (again, Gaussian distribution is assumed to infer the $90 \%$ C.L. from $1 \sigma$ range), which lie below the $\alpha$ band from Ref. [60]. This reconfirms the low LY of nuclear recoil compared with $\alpha$ 's. Conservatively, we assume the events below the purple $\alpha$ band as dark matter candidates, where three such events are found for the phonon energy between 300 and $2000 \mathrm{keV}$. We do not include the events between 1800 and $2000 \mathrm{keV}$, as they are attributable to ${ }^{144} \mathrm{Nd}$ decays and are far away from the W LY, which dominates the dark matter scattering at high recoil energy due to kinematical suppression.

We also show the event selection in the $\mathrm{PbWO}_{4}$ experiment [61] in Fig. 7. The LY of $\alpha$ 's is given in Eq. (15). We show the $1 \sigma$ range of the corresponding LY with blue lines. To be conservative, we assume events below the $1 \sigma \alpha$ band to be dark matter candidates, where 12 such events are identified.
[1] A. H. Abdelhameed et al. (CRESST Collaboration), First results from the CRESST-III low-mass dark matter program, Phys. Rev. D 100, 102002 (2019).

[2] Z.Z. Liu et al. (CDEX Collaboration), Constraints on Spin-Independent Nucleus Scattering with sub-GeV Weakly Interacting Massive Particle Dark Matter from the CDEX-1B Experiment at the China Jinping Underground Laboratory, Phys. Rev. Lett. 123, 161301 (2019).

[3] E. Armengaud et al. (EDELWEISS Collaboration), Searching for low-mass dark matter particles with a massive Ge bolometer operated above-ground, Phys. Rev. D 99, 082003 (2019).

[4] D. S. Akerib et al. (LUX Collaboration), Results of a Search for Sub-GeV Dark Matter Using 2013 LUX Data, Phys. Rev. Lett. 122, 131301 (2019).

[5] E. Aprile et al. (XENON Collaboration), Search for Light Dark Matter Interactions Enhanced by the Migdal Effect or Bremsstrahlung in XENON1T, Phys. Rev. Lett. 123, 241803 (2019).

[6] E. Aprile et al. (XENON Collaboration), Light Dark Matter Search with Ionization Signals in XENON1T, Phys. Rev. Lett. 123, 251801 (2019).

[7] P. Agnes et al. (DarkSide Collaboration), Constraints on Sub-GeV Dark-Matter-Electron Scattering from the DarkSide-50 Experiment, Phys. Rev. Lett. 121, 111303 (2018).

[8] Chen Cheng et al. (PandaX-II Collaboration), Search for Light Dark Matter-Electron Scatterings in the PandaX-II Experiment, Phys. Rev. Lett. 126, 211803 (2021).

[9] Liron Barak et al. (SENSEI Collaboration), SENSEI: Direct-Detection Results on sub-GeV Dark Matter from a New Skipper-CCD, Phys. Rev. Lett. 125, 171802 (2020).

[10] A. Aguilar-Arevalo et al. (DAMIC Collaboration), Constraints on Light Dark Matter Particles Interacting with Electrons from DAMIC at SNOLAB, Phys. Rev. Lett. 123, 181802 (2019).

[11] Q. Arnaud et al. (EDELWEISS Collaboration), First Germanium-Based Constraints on sub-MeV Dark Matter with the EDELWEISS Experiment, Phys. Rev. Lett. 125, 141301 (2020).

[12] D. W. Amaral et al. (SuperCDMS Collaboration), Constraints on low-mass, relic dark matter candidates from a surface-operated SuperCDMS single-charge sensitive detector, Phys. Rev. D 102, 091101 (2020).
[13] Amit Bhoonah, Joseph Bramante, Fatemeh Elahi, and Sarah Schon, Galactic Center gas clouds and novel bounds on ultralight dark photon, vector portal, strongly interacting, composite, and super-heavy dark matter, Phys. Rev. D 100, 023001 (2019).

[14] Joseph Bramante, Andrew Buchanan, Alan Goodman, and Eesha Lodhi, Terrestrial and martian heat flow limits on dark matter, Phys. Rev. D 101, 043001 (2020).

[15] Raghuveer Garani and Peter Tinyakov, Constraints on dark matter from the moon, Phys. Lett. B 804, 135403 (2020).

[16] Yang Bai, Mrunal Korwar, and Nicholas Orlofsky, Electroweak-symmetric dark monopoles from preheating, J. High Energy Phys. 07 (2020) 167.

[17] Christopher V. Cappiello, J. I. Collar, and John F. Beacom, New experimental constraints in a new landscape for composite dark matter, Phys. Rev. D 103, 023019 (2021).

[18] Javier F. Acevedo, Joseph Bramante, Alan Goodman, Joachim Kopp, and Toby Opferkuch, Dark matter, destroyer of worlds: Neutrino, thermal, and existential signatures from black holes in the sun and earth, J. Cosmol. Astropart. Phys. 04 (2021) 026.

[19] Amit Bhoonah, Joseph Bramante, Brian Courtman, and Ningqiang Song, Etching plastic searches for dark matter, Phys. Rev. D 103, 103001 (2021).

[20] Jagjit Singh Sidhu, Robert J. Scherrer, and Glenn Starkman, Death and serious injury from dark matter, Phys. Lett. B 803, 135300 (2020).

[21] Jagjit Singh Sidhu and Glenn Starkman, Macroscopic dark matter constraints from bolide camera networks, Phys. Rev. D 100, 123008 (2019).

[22] Jagjit Singh Sidhu, Charge constraints of macroscopic dark matter, Phys. Rev. D 101, 043526 (2020).

[23] Yang Bai, Andrew J. Long, and Sida Lu, Dark quark nuggets, Phys. Rev. D 99, 055047 (2019).

[24] Lawrence J. Hall, Takeo Moroi, and Hitoshi Murayama, Sneutrino cold dark matter with lepton number violation, Phys. Lett. B 424, 305 (1998).

[25] David Tucker-Smith and Neal Weiner, Inelastic dark matter, Phys. Rev. D 64, 043502 (2001).

[26] David Tucker-Smith and Neal Weiner, The status of inelastic dark matter, Phys. Rev. D 72, 063509 (2005). 
[27] Chiara Arina and Nicolao Fornengo, Sneutrino cold dark matter, a new analysis: Relic abundance and detection rates, J. High Energy Phys. 11 (2007) 029.

[28] Yanou Cui, David E. Morrissey, David Poland, and Lisa Randall, Candidates for inelastic dark matter, J. High Energy Phys. 05 (2009) 076.

[29] Patrick J. Fox, Graham D. Kribs, and Tim M. P. Tait, Interpreting dark matter direct detection independently of the local velocity and density distribution, Phys. Rev. D 83, 034007 (2011).

[30] Haipeng An, P. S. Bhupal Dev, Yi Cai, and R. N. Mohapatra, Sneutrino Dark Matter in Gauged Inverse Seesaw Models for Neutrinos, Phys. Rev. Lett. 108, 081806 (2012).

[31] Maxim Pospelov, Neal Weiner, and Itay Yavin, Dark matter detection in two easy steps, Phys. Rev. D 89, 055008 (2014).

[32] Keith R. Dienes, Jason Kumar, Brooks Thomas, and David Yaylali, Dark-Matter Decay as a Complementary Probe of Multicomponent Dark Sectors, Phys. Rev. Lett. 114, 051301 (2015).

[33] G. Barello, Spencer Chang, and Christopher A. Newby, A model independent approach to inelastic dark matter scattering, Phys. Rev. D 90, 094027 (2014).

[34] Douglas P. Finkbeiner and Neal Weiner, Exciting dark matter and the INTEGRAL/SPI $511 \mathrm{keV}$ signal, Phys. Rev. D 76, 083519 (2007).

[35] Nanda Rea, Roberto Turolla, Silvia Zane, Andrea Tramacere, Luigi Stella, and Gianluca Israel, Spectral modelling of the high energy emission of the magnetar 4U 0142 + 614, Astrophys. J. Lett. 661, L65 (2007).

[36] James M. Cline, Andrew R. Frey, and Fang Chen, Metastable dark matter mechanisms for INTEGRAL $511 \mathrm{keV} \gamma$ rays and DAMA/CoGeNT events, Phys. Rev. D 83, 083511 (2011).

[37] J. M. Cline and A. R. Frey, Abelian dark matter models for $511 \mathrm{keV}$ gamma rays and direct detection, Ann. Phys. (Amsterdam) 524, 579 (2012).

[38] Yohei Ema, Filippo Sala, and Ryosuke Sato, Dark matter models for the $511 \mathrm{keV}$ galactic line predict keV electron recoils on Earth, Eur. Phys. J. C 81, 129 (2021).

[39] Spencer Chang, Graham D. Kribs, David Tucker-Smith, and Neal Weiner, Inelastic dark matter in light of DAMA/ LIBRA, Phys. Rev. D 79, 043513 (2009).

[40] David Tucker-Smith and Neal Weiner, Inelastic dark matter at DAMA, CDMS and future experiments, Nucl. Phys. B, Proc. Suppl. 124, 197 (2003).

[41] Sunghyun Kang, S. Scopel, and Gaurav Tomar, Probing DAMA/LIBRA data in the full parameter space of WIMP effective models of inelastic scattering, Phys. Rev. D 99, 103019 (2019).

[42] Sunniva Jacobsen, Katherine Freese, Chris Kelso, Pearl Sandick, and Patrick Stengel, Inelastic dark matter scattering off Thallium cannot save DAMA, J. Cosmol. Astropart. Phys. 10 (2021) 070.

[43] E. Aprile et al. (XENON Collaboration), Excess electronic recoil events in XENON1T, Phys. Rev. D 102, 072004 (2020).

[44] Joseph Bramante and Ningqiang Song, Electric But Not Eclectic: Thermal Relic Dark Matter for the XENON1T Excess, Phys. Rev. Lett. 125, 161805 (2020).
[45] Keisuke Harigaya, Yuichiro Nakai, and Motoo Suzuki, Inelastic dark matter electron scattering and the XENON1T excess, Phys. Lett. B 809, 135729 (2020).

[46] Debasish Borah, Satyabrata Mahapatra, Dibyendu Nanda, and Narendra Sahu, Inelastic fermion dark matter origin of XENON1T excess with muon $(g-2)$ and light neutrino mass, Phys. Lett. B 811, 135933 (2020).

[47] Debajyoti Choudhury, Suvam Maharana, Divya Sachdeva, and Vandana Sahdev, Dark matter, muon anomalous magnetic moment, and the XENON1T excess, Phys. Rev. D 103, 015006 (2021).

[48] Debasish Borah, Satyabrata Mahapatra, and Narendra Sahu, Connecting low scale seesaw for neutrino mass and inelastic sub-GeV dark matter with abelian gauge symmetry, Nucl. Phys. B968, 115407 (2021).

[49] Amin Aboubrahim, Michael Klasen, and Pran Nath, Xenon-1T excess as a possible signal of a sub-GeV hidden sector dark matter, J. High Energy Phys. 02 (2021) 229.

[50] Hyun Min Lee, Exothermic dark matter for XENON1T excess, J. High Energy Phys. 01 (2021) 019.

[51] Seungwon Baek, Jongkuk Kim, and P. Ko, XENON1T excess in local $Z_{2}$ DM models with light dark sector, Phys. Lett. B 810, 135848 (2020).

[52] Masha Baryakhtar, Asher Berlin, Hongwan Liu, and Neal Weiner, Electromagnetic signals of inelastic dark matter scattering, arXiv:2006.13918.

[53] Wei Chao, Yu Gao, and Ming jie Jin, Pseudo-dirac dark matter in XENON1T, arXiv:2006.16145.

[54] Haipeng An and Daneng Yang, Direct detection of freezein inelastic dark matter, Phys. Lett. B 818, 136408 (2021).

[55] Zidu Lin, Matthew E. Caplan, Charles J. Horowitz, and Cecilia Lunardini, Fast neutrino cooling of nuclear pasta in neutron stars: Molecular dynamics simulations, Phys. Rev. C 102, 045801 (2020).

[56] Wai-Yee Keung, Danny Marfatia, and Po-Yan Tseng, Stellar cooling, inelastic dark matter, and XENON, JHEAp 30, 9 (2021).

[57] Hong-Jian He, Yu-Chen Wang, and Jiaming Zheng, GeV scale inelastic dark matter with dark photon mediator via direct detection and cosmological/laboratory constraints, arXiv:2012.05891.

[58] Soo-Min Choi, Hyun Min Lee, and Bin Zhu, Exothermic dark mesons in light of electron recoil excess at XENON1T, J. High Energy Phys. 04 (2021) 251.

[59] Manoranjan Dutta, Satyabrata Mahapatra, Debasish Borah, and Narendra Sahu, Self-interacting inelastic dark matter in the light of XENON1T excess, Phys. Rev. D 103, 095018 (2021).

[60] A. Münster et al., Radiopurity of $\mathrm{CaWO}_{4}$ crystals for direct dark matter search with CRESST and EURECA, J. Cosmol. Astropart. Phys. 05 (2014) 018.

[61] J. W. Beeman et al., New experimental limits on the alpha decays of lead isotopes, Eur. Phys. J. A 49, 50 (2013).

[62] Maxim Pospelov, Surjeet Rajendran, and Harikrishnan Ramani, Metastable nuclear isomers as dark matter accelerators, Phys. Rev. D 101, 055001 (2020).

[63] Björn Lehnert, Harikrishnan Ramani, Mikael Hult, Guillaume Lutter, Maxim Pospelov, Surjeet Rajendran, and Kai Zuber, Search for Dark Matter Induced deExcitation of ${ }^{180 m}$ ta, Phys. Rev. Lett. 124, 181802 (2020). 
[64] L. Baudis, G. Kessler, P. Klos, R. F. Lang, J. Menéndez, S. Reichard, and A. Schwenk, Signatures of dark matter scattering inelastically off nuclei, Phys. Rev. D 88, 115014 (2013).

[65] Christopher McCabe, Prospects for dark matter detection with inelastic transitions of xenon, J. Cosmol. Astropart. Phys. 05 (2016) 033.

[66] E. Aprile et al. (XENON Collaboration), Search for inelastic scattering of WIMP dark matter in XENON1T, Phys. Rev. D 103, 063028 (2021).

[67] B. Broerman, M. Laubenstein, S. Nagorny, N. Song, and A. C. Vincent, A search for rare and induced nuclear decays in hafnium, Nucl. Phys. A1012, 122212 (2021).

[68] F. G. Kondev, Nuclear data sheets for $A=177$, Nucl. Data Sheets 98, 801 (2003).

[69] E. Achterberg, O. A. Capurro, and G. V. Marti, Nuclear data sheets for $A=178$, Nucl. Data Sheets 110, 1473 (2009).

[70] E. A. McCutchan, Nuclear data sheets for $A=180$, Nucl. Data Sheets 126, 151 (2015).

[71] T. D. Johnson and Balraj Singh, Nuclear data sheets for $A=189$, Nucl. Data Sheets 142, 1 (2017).

[72] F. G. Kondev, Nuclear data sheets for $A=201$, Nucl. Data Sheets 108, 365 (2007).

[73] Brian Batell, Maxim Pospelov, and Adam Ritz, Direct detection of multi-component secluded WIMPs, Phys. Rev. D 79, 115019 (2009).

[74] Giacomo Monari, Benoit Famaey, Ismael Carrillo, Tilmann Piffl, Matthias Steinmetz, Rosemary F. G. Wyse, Friedrich Anders, Cristina Chiappini, and Katja Janssen, The escape speed curve of the galaxy obtained from Gaia DR2 implies a heavy Milky Way, Astron. Astrophys. 616, L9 (2018).

[75] Til Piffl et al., The RAVE survey: The Galactic escape speed and the mass of the Milky Way, Astron. Astrophys. 562, A91 (2014).

[76] Angus A Williams, Vasily Belokurov, Andrew R Casey, and $\mathrm{N}$ Wyn Evans, On the run: Mapping the escape speed across the galaxy with sdss, Mon. Not. R. Astron. Soc. 468, 2359 (2017).

[77] Alis J Deason, Azadeh Fattahi, Vasily Belokurov, N. Wyn Evans, Robert J. J. Grand, Federico Marinacci, and Rüdiger Pakmor, The local high-velocity tail and the galactic escape speed, Mon. Not. R. Astron. Soc. 485, 3514 (2019).

[78] F. Petricca et al. (CRESST Collaboration), First results on low-mass dark matter from the CRESST-III experiment, J. Phys. Conf. Ser. 1342, 012076 (2019).

[79] G. Angloher et al. (CRESST Collaboration), Results on light dark matter particles with a low-threshold CRESST-II detector, Eur. Phys. J. C 76, 25 (2016).

[80] J. Engel, S. Pittel, and P. Vogel, Nuclear physics of dark matter detection, Int. J. Mod. Phys. E 01, 1 (1992).

[81] J. Engel and P. Vogel, Neutralino inelastic scattering with subsequent detection of nuclear gamma-rays, Phys. Rev. D 61, 063503 (2000).

[82] Coral M. Baglin, Nuclear data sheets for $A=169$, Nucl. Data Sheets 109, 2033 (2008).

[83] Jouni Suhonen, From Nucleons to Nucleus, Theoretical and Mathematical Physics (Springer, Berlin, 2007).
[84] G. Angloher et al. (CRESST Collaboration), Description of CRESST-II data, arXiv:1701.08157.

[85] E. Bertoldo et al. (CRESST Collaboration), Lithiumcontaining crystals for light dark matter search experiments, J. Low Temp. Phys. 199, 510 (2020).

[86] G. Angloher et al., Results from $730 \mathrm{~kg}$ days of the CRESST-II Dark Matter Search, Eur. Phys. J. C 72, 1971 (2012).

[87] P. Belli et al., Search for $\alpha$ decay of naturally occurring osmium nuclides accompanied by $\gamma$ quanta, Phys. Rev. C 102, 024605 (2020).

[88] Mohsen B Challan, Gamma-ray efficiency of a hpge detector as a function of energy and geometry, Appl. Radiat. Isot. 82, 166 (2013).

[89] Lucas Bodenstein-Dresler et al. (COBRA Collaboration), Quenching of $g_{\mathrm{A}}$ deduced from the $\beta$-spectrum shape of ${ }^{113} \mathrm{Cd}$ measured with the COBRA experiment, Phys. Lett. B 800, 135092 (2020).

[90] V. Novati et al., Charge-to-heat transducers exploiting the Neganov-Trofimov-Luke effect for light detection in rareevent searches, Nucl. Instrum. Methods Phys. Res., Sect. A 940, 320 (2019).

[91] J. W. Beeman et al., Characterization of bolometric light detectors for rare event searches, J. Instrum. 8, P07021 (2013).

[92] M. Barucci, J. W. Beeman, V. Caracciolo, L. Pagnanini, L. Pattavina, G. Pessina, S. Pirro, C. Rusconi, and K. Schäffner, Cryogenic light detectors with enhanced performance for rare event physics, Nucl. Instrum. Methods Phys. Res., Sect. A 935, 150 (2019).

[93] C. Amole et al. (PICO Collaboration), Dark matter search results from the PICO-60 $\mathrm{CF}_{3} \mathrm{I}$ bubble chamber, Phys. Rev. D 93, 052014 (2016).

[94] E. Aprile et al. (XENON Collaboration), Dark Matter Search Results from a One Ton-Year Exposure of XENON1T, Phys. Rev. Lett. 121, 111302 (2018).

[95] XENON1T-2018, https://github.com/bradkav/Xenon1T2018 (2018).

[96] Joseph Bramante, Patrick J. Fox, Graham D. Kribs, and Adam Martin, Inelastic frontier: Discovering dark matter at high recoil energy, Phys. Rev. D 94, 115026 (2016).

[97] Torsten Bringmann and Maxim Pospelov, Novel Direct Detection Constraints on Light Dark Matter, Phys. Rev. Lett. 122, 171801 (2019).

[98] Christopher V. Cappiello, Kenny C. Y. Ng, and John F. Beacom, Reverse direct detection: Cosmic ray scattering with light dark matter, Phys. Rev. D 99, 063004 (2019).

[99] Christopher Cappiello and John F. Beacom, Strong new limits on light dark matter from neutrino experiments, Phys. Rev. D 100, 103011 (2019).

[100] Wonsub Cho, Ki-Young Choi, and Seong Moon Yoo, Searching for boosted dark matter mediated by a new gauge boson, Phys. Rev. D 102, 095010 (2020).

[101] Wen Yin, Highly-boosted dark matter and cutoff for cosmic-ray neutrinos through neutrino portal, EPJ Web Conf. 208, 04003 (2019).

[102] Yongsoo Jho, Jong-Chul Park, Seong Chan Park, and Po-Yan Tseng, Cosmic-neutrino-boosted dark matter ( $\nu \mathrm{BDM})$, arXiv:2101.11262. 
[103] Anirban Das and Manibrata Sen, Boosted dark matter from diffuse supernova neutrinos, Phys. Rev. D 104, 075029 (2021).

[104] Timon Emken, Solar reflection of light dark matter with heavy mediators, arXiv:2102.12483.

[105] Kaustubh Agashe, Yanou Cui, Lina Necib, and Jesse Thaler, (In)direct detection of boosted dark matter, J. Cosmol. Astropart. Phys. 10 (2014) 062.

[106] Joshua Berger, Yanou Cui, and Yue Zhao, Detecting boosted dark matter from the sun with large volume neutrino detectors, J. Cosmol. Astropart. Phys. 02 (2015) 005.

[107] Francesco D'Eramo and Jesse Thaler, Semi-annihilation of dark matter, J. High Energy Phys. 06 (2010) 109.

[108] Juri Smirnov and John F. Beacom, New Freezeout Mechanism for Strongly Interacting Dark Matter, Phys. Rev. Lett. 125, 131301 (2020).

[109] Kyoungchul Kong, Gopolang Mohlabeng, and Jong-Chul Park, Boosted dark matter signals uplifted with selfinteraction, Phys. Lett. B 743, 256 (2015).

[110] Gian F. Giudice, Doojin Kim, Jong-Chul Park, and Seodong Shin, Inelastic boosted dark matter at direct detection experiments, Phys. Lett. B 780, 543 (2018).

[111] Nicole F. Bell, James B. Dent, Bhaskar Dutta, Sumit Ghosh, Jason Kumar, Jayden L. Newstead, and Ian M.
Shoemaker, Cosmic-ray upscattered inelastic dark matter, Phys. Rev. D 104, 076020 (2021).

[112] L. Pattavina, S. Nagorny, S. Nisi, L. Pagnanini, G. Pessina, S. Pirro, C. Rusconi, K. Schäffner, V. N. Shlegel, and V. N. Zhdankov, Production and characterisation of a $\mathrm{PbMoO}_{4}$ cryogenic detector from archaeological Pb, Eur. Phys. J. A 56, 38 (2020).

[113] L. Pattavina et al. (RES-NOVA Collaboration), RES-NOVA sensitivity to core-collapse and failed core-collapse supernova neutrinos, J. Cosmol. Astropart. Phys. 10 (2021) 064.

[114] E. Browne and Huo Junde, Nuclear data sheets for $A=174$, Nucl. Data Sheets 87, 15 (1999).

[115] M. S. Basunia, Nuclear data sheets for $A=176$, Nucl. Data Sheets 107, 791 (2006).

[116] Coral M. Baglin, Nuclear data sheets for $A=179$, Nucl. Data Sheets 110, 265 (2009).

[117] M. S. Basunia, Nuclear data sheets for $A=187$, Nucl. Data Sheets 110, 999 (2009).

[118] R. Strauss, G. Angloher, A. Bento, C. Bucci, L. Canonica, Walter Carli, A. Erb, F. von Feilitzsch, P. Gorla, A. Gütlein et al., Energy-dependent light quenching in cawo $_{4}$ crystals at $\mathrm{mK}$ temperatures, Eur. Phys. J. C 74, 2957 (2014). 